\title{
CAMBIOS EN LA INDUSTRIA DE LOS SUPERMERCADOS CONCENTRACIÓN, HIPERMERCADOS, RELACIONES CON PROVEEDORES Y MARCAS PROPIAS*
}

\section{Loreto Lira}

A partir de la década de los noventa se comienza a producir en el mundo un cambio estructural en la industria de los supermercados, caracterizado principalmente por una creciente concentración de mercado, un fuerte auge de los hipermercados y una sostenida política de precios bajos para los consumidores.

El objetivo de este artículo es analizar las razones que están detrás de los cambios de esta industria y algunos de los efectos que se han derivado de dichos cambios, basado tanto en consideraciones teóricas como en la experiencia internacional. En particular se analizan los efectos en las relaciones proveedores-distribuidores y sus consecuencias sobre los consumidores finales, así como el impacto de la nueva organización industrial en el surgimiento de las marcas propias.

LORETO LIRA. Profesora de la Facultad de Ciencias Económicas y Empresariales, Universidad de los Andes, Santiago.

* La autora agradece los comentarios de Carlos Antonio Díaz. 


\section{Introducción}

A

partir de la década de los noventa se comienza a producir en el mundo un cambio estructural en la industria de los supermercados, caracterizada principalmente por una creciente concentración de mercado, un fuerte auge de los hipermercados y una sostenida política de precios bajos para los consumidores.

Tras estos cambios se identifican claramente diversos factores. Por el lado de la oferta, los avances tecnológicos en la información y comunicaciones crean economías de escala y de ámbito que, por una parte, permiten la expansión de cadenas de hipermercados (muy intensivas en el uso de la tecnología de la información) y, por otra, llevan a la concentración de la industria. Por el lado de la demanda, el recurso tiempo se hace cada vez más escaso debido a la creciente participación de la mujer en el mercado laboral, lo que aumenta las preferencias por los hipermercados, pues dan la posibilidad de comprar un mayor número de productos en un mismo lugar.

El objetivo de este artículo es analizar en profundidad las razones que están detrás de los cambios en esta industria y algunos de los efectos que se han derivado de dichos cambios, basado tanto en consideraciones teóricas como en la experiencia internacional. En particular se analizan los efectos en las relaciones proveedores-distribuidores y sus consecuencias sobre los consumidores finales. Debe destacarse que la relación entre proveedores y distribuidores es quizás uno de los aspectos más discutidos recientemente tanto en el mundo como en Chile en referencia a este tema. Asimismo, se analiza el impacto de la nueva organización industrial en el surgimiento de las marcas propias.

Desde un punto de vista teórico, se podría sostener que la concentración de la industria de la distribución desplaza el poder de negociación desde los proveedores hacia los distribuidores. El impacto que esto tenga sobre los consumidores de los bienes finales dependerá de la forma en que opere la industria de la distribución. En caso de que las cadenas de la distribución compitan a través de los precios, los beneficios que puedan obtener en sus negociaciones con los proveedores, como consecuencia del desplazamiento de poder, serán traspasadas a los consumidores. En tales circunstancias, estos últimos son los principales beneficiados. Una de las preguntas que cabe hacerse es entonces si se ha producido dicho desplazamiento de poder de negociación. La evidencia empírica internacional no permite afirmar que así haya sido, medido como la evolución relativa de diversos índices de rentabilidad de proveedores y distribuidores. Por el contrario, hay cierta evidencia de que, en algunas categorías de productos, 
los proveedores incluso habrían mejorado sus rentabilidades relativas a los distribuidores. Entre las posibles explicaciones de estos hallazgos podría mencionarse el hecho de que los proveedores o productores han aumentado la eficiencia de sus negocios; otra es que estos últimos también han tendido a concentrarse $\mathrm{y}$, por último, podría decirse que los distribuidores no han sido capaces de retener los beneficios de las negociaciones, puesto que han debido traspasarlos a los consumidores.

Otra consecuencia de la concentración de la industria de los supermercados es el auge experimentado por las marcas propias. Éstas se han transformado en una pieza fundamental del negocio. Se identifican tres razones principales: son una fuente directa de ingresos, aportan a la diferenciación de las cadenas de supermercados entre sí y cooperan en el desplazamiento de poderes desde los proveedores hacia los distribuidores. En este auge también influye el hecho de que los consumidores actuales son menos leales a las marcas tradicionales y más racionales en sus compras, ateniéndose principalmente a las variables de calidad y precio.

Este trabajo se organiza de la siguiente manera: la sección 2 describe las principales tendencias de la industria de los supermercados y sus causas. La sección 3 presenta los casos de Wal-Mart, Tesco y D\&S. La sección 4 se centra en las implicancias de estos cambios en las relaciones entre proveedores y distribuidores. En la sección 5 se investiga el impacto que ha tenido la nueva organización de la industria en el desarrollo de marcas propias. En la sección 6 se presentan conclusiones.

\section{Las principales tendencias en la industria de los supermercados y sus causas}

En los últimos años han ocurrido profundos cambios en la industria de los supermercados en el mundo, cuyas principales tendencias son: la creciente concentración de la industria, políticas de precios bajos y el incremento de los hipermercados. Existen causas de oferta y de demanda tras estos cambios.

\subsection{Creciente concentración de la industria}

La industria de los supermercados se ha ido concentrando en el mundo en los últimos años. Así, en Estados Unidos, en el período 19871997, las ventas brutas del sector comercio crecieron en términos reales $35,4 \%$, mientras que el número de establecimientos creció durante ese 
mismo período sólo 6,7\%. Adicionalmente, la participación en el mercado de las 50 empresas más grandes pasó de 20,3\% en 1987 a 25,7\% en 1997 (Sieling, Friedman y Dumas, 2001). En Europa también se da esta tendencia: las diez principales cadenas de distribución que tenían el $28 \%$ de participación en el mercado en 1992 alcanzaron el 36\% en 1997. El número de locales de venta de abarrotes cayó de más de 140.000 a menos de 40.000 en el período 1960-97 en Gran Bretaña, mientras que en Francia bajó de 152.000 a 41.700 entre 1968-94, y en la antigua Alemania Occidental de 212.700 a 69.000 en el período 1955-89 (Dobson y Waterson, 1999). En Chile se observa algo similar, aunque con un cierto desfase en el tiempo: las cuatro principales cadenas de supermercados aumentaron su participación en el mercado desde 47,3\% en 1996 hasta 62,1\% en mayo de 2004 (Asach).

\section{Causas de la concentración}

La concentración de la industria de los supermercados es una consecuencia directa del surgimiento de economías de escala y de ámbito a partir de fines de la década de los ochenta en el mundo. Éstas generan fuertes incentivos para que las empresas aumenten el tamaño de su negocio tanto en el volumen como en la variedad de los productos ofrecidos, ya que de esta forma logran reducir los costos medios de su gestión, es decir, incrementan la eficiencia de su operación (Dobson y Waterson, 1997). A su vez, las economías de escala y de ámbito encuentran su origen en los avances tecnológicos en el área de la informática y de las comunicaciones, los cuales básicamente han estado orientados a aumentar la eficiencia en el manejo de los inventarios, pieza clave en el negocio del retail. Específicamente, los avances tecnológicos han permitido disminuir los períodos de mantención de mercadería en stock y acortar los plazos entre la medición del nivel de inventarios y el despacho de nuevas órdenes. Esto se ha logrado a través de la implementación de los sistemas interconectados de transmisión de datos (EDI: electronic data interchange), que mantienen en comunicación computacional permanente a distribuidores con proveedores, y del uso de los códigos de barras, que hace posible llevar mediciones más actualizadas de los inventarios (Holmes 2001). Nakamura (1997) define como "revolución del retail" a la rápida automatización de los procesos de las transacciones en este sector.

Los estudios de Fernald y Ramnath (2004) investigan la productividad total de los factores en Estados Unidos durante la década de los noven- 
ta para todos los sectores de la economía. Ellos evidencian un aceleramiento general en la productividad total de los factores (PTF) en Estados Unidos a partir de la segunda mitad de la década de los noventa. Entre ellos se destacan los sectores de ventas al por mayor y retail, ya que exhiben el mayor crecimiento en la PTF a partir de 1995. En el período 1995 a 2000, esta tasa creció a $5,33 \%$ y la del comercio mayorista a 5,37\% versus el $2,08 \%$ del promedio de la economía no agrícola. Ver Cuadro $\mathrm{N}^{\circ} 1$.

CUADRO N ${ }^{\circ}$ : $\quad$ ESTADOS UNIDOS PERÍODO 1990-2000: CRECIMIENTO DE LA PRODUCTIVIDAD TOTAL DE LOS FACTORES (PTF)

(Tasa porcentual anual)

\begin{tabular}{lcr}
\hline & $1990-1994$ & $1995-2000$ \\
\hline Minería & 3,08 & $-2,15$ \\
Industria & 2,40 & 2,76 \\
Construcción & 0,39 & $-0,98$ \\
Transporte & 1,69 & 1,53 \\
Comunicaciones & 2,31 & 0,15 \\
Electricidad, gas y agua & 0,42 & 0,17 \\
Comercio mayorista & $\mathbf{1 , 6 6}$ & $\mathbf{5 , 3 7}$ \\
Comercio minorista & $\mathbf{0 , 8 3}$ & $\mathbf{5 , 3 3}$ \\
Sector financiero & 0,44 & 3,39 \\
Sector inmobiliario & 1,12 & 0,40 \\
Otros servicios & $-1,89$ & 0,08 \\
Total sector privado no agrícola & 0,91 & 2,88 \\
\hline
\end{tabular}

Fuente: Economic Perspectives, 2004, Vol. 28, № 1.

Fernald y Ramnath señalan que los sectores de la economía más intensivos en el uso de la tecnología de la información (TI) son los que presentan una mayor PTF. Dado que los sectores del comercio detallista y mayorista son efectivamente los que más aumentaron su PTF, se deduce que ellos deben ser los más intensivos en el uso de la tecnología de la información.

La productividad del trabajo también ha crecido fuertemente en el sector retail. Se estima que ésta se aceleró bruscamente hacia fines de los noventa en Estados Unidos. Durante el período 1995-1999, la productividad del trabajo en el sector comercio creció a una tasa promedio anual de $3,1 \%$, casi el doble de la tasa de crecimiento de esta variable en la economía en general en el período 1990-1995 (1,6\%). La principal causa de esta mayor productividad se encuentra en los adelantos tecnológicos computacionales que permiten mejores manejos de inventarios desde el punto de 
venta, ajustar precios de forma más eficiente y el desarrollo de bases de datos de los clientes (Sieling et al., 2001).

\subsection{Políticas de precios bajos}

Las principales distribuidoras han ido implementando la política de precios bajos. Wal-Mart ha sido la cadena de distribución que ha liderado esta política en el mundo. Algunos estudios internacionales estiman que Wal-Mart en Estados Unidos vende sus productos en promedio a precios $25 \%$ inferiores a los de la competencia (véase Cuadro $\mathrm{N}^{\circ} 3$ ). En Chile, también ha estado presente la venta a precios bajos, sobre todo a partir de la segunda mitad de los noventa. Esta política ha sido llevada a cabo principalmente por $\mathrm{D} \& \mathrm{~S}$, la mayor distribuidora nacional. La creciente competitividad en precios de los productos vendidos por los supermercados puede nombrarse como una de las causas que han contribuido a disminuir la inflación en Chile, especialmente en los últimos cinco años. El Cuadro $\mathrm{N}^{\circ} 2$ muestra las variaciones porcentuales del IPC a partir de 1994, en forma separada en dos períodos (1994-1998 y 1999-julio de 2004). Se ve que en el segundo período los rubros susceptibles de ser vendidos por supermercados han tenido variaciones de precios muy inferiores al promedio del IPC. En efecto, el IPC ha subido 16,4\%, en tanto que los alimentos han aumentado sólo en $4,7 \%$, el equipamiento de la vivienda ha bajado $6,5 \%$ y el vestuario ha descendido en $23,9 \%$.

CUADRO N$^{\circ}$ 2: $\quad$ VARIACIÓN PORCENTUAL DEL IPC POR RUBRO

\begin{tabular}{lccc}
\hline & $1994-2004$ jul & $1994-1998$ & $1999-2004$ jul \\
\hline IPC & 62,0 & 39,5 & 16,1 \\
& & & \\
Alimentación & 39,8 & 33,5 & 4,7 \\
Vivienda & 78,7 & 40,8 & 26,9 \\
Equip. de la vivienda & 18,3 & 26,5 & $-6,5$ \\
Vestuario & $-35,0$ & $-14,5$ & $-23,9$ \\
Transporte & 126,9 & 47,2 & 54,1 \\
Salud & 106,2 & 61,3 & 27,9 \\
Educación y recreación & 126,4 & 80,6 & $-4,6$ \\
Otros & 47,8 & 54,9 & \\
& & & \\
\hline
\end{tabular}

Fuente: INE (Instituto Nacional de Estadísticas): www.ine.cl 


\section{Causas de los precios bajos}

La concentración de la industria de los supermercados se ha ido produciendo a través de la competencia en los precios. Los distribuidores que han sido capaces de incorporar anticipadamente los avances tecnológicos en su negocio han podido ofrecer sus productos a precios menores que los de sus competidores, debido a la reducción en sus costos operativos que dichos cambios significan. De esta forma, las cadenas de supermercados, con una estructura de costos inferiores y, por lo tanto, con mayor utilización de la tecnología, han ido desplazando al resto de los distribuidores, lo que ha redundado en una mayor concentración de la industria. Esta sustitución de empresas menos eficientes por las más eficientes ha ido generando un aumento en la productividad total del sector retail (Foster, Haltiwanger y Krizan, 2002).

\subsection{Incremento de los hipermercados}

En la actualidad la industria de supermercados contempla al menos tres formatos de venta, que varían de acuerdo con el tamaño de sus salas de venta. El más chico se refiere a los supermercados con salas de venta de hasta 3.000 metros cuadrados, después vienen los supermercados tradicionales con locales de venta de hasta 6.000 metros cuadrados y por último, los hipermercados con locales de venta sobre los 6.000 metros cuadrados, donde se ofrece, además de los productos tradicionales, una serie de otras categorías, como electrodomésticos, electrónica, librería, jardinería, piscinería, vestuario, zapatería, juguetería y artículos de decoración. En estos formatos los hipermercados han sido los que más se han desarrollado en los últimos años. Es así como en Estados Unidos el gasto total en comida de los consumidores en los supermercados tradicionales cayó desde 42,8\% en 1988 hasta $13,4 \%$ en 1998 y el número de supermercados tradicionales se redujo en un 8\% entre 1987 y 1997 (Sieling et al., 2001). Messinger y Narasimhan (1995) destacan que el número de ítems vendidos por local aumentó de 7.800 en 1970 a 18.466 en 1993. El tamaño promedio de los locales también creció desde $2.508 \mathrm{~m}^{2}$ en 1983 hasta $3.456 \mathrm{~m}^{2}$ en 1993 . La participación de las ventas de los supermercados convencionales se redujo de $73,1 \%$ en 1980 a 30,4\% en 1991. En Chile, en el rubro supermercados, el porcentaje de ventas de los supermercados tradicionales cayó desde $71 \%$ hasta $65 \%$ en el período 2000-2003. Como contrapartida, aumentaron las ventas de los hipermercados de $26 \%$ a $32 \%$, manteniéndose constante las 
de los autoservicios, que corresponden, de acuerdo con la definición, a locales de conveniencia (locales cuya superficie de venta es inferior a los 1.000 metros cuadrados) (Nielsen 2004).

\section{Causas de oferta en el incremento de los hipermercados}

Holmes (2001) afirma que los cambios en la tecnología de la información aumentan el tamaño óptimo de local, lo que es coherente con el éxito de los formatos de hipermercados. Señala que los avances tecnológicos, como los códigos de barras, y los sistemas de comunicaciones con proveedores permiten, por una parte, obtener mediciones más rápidas de los inventarios en los locales de venta y, por otra, aumentar la velocidad de las nuevas órdenes de mercaderías. Así se disminuye el tiempo que transcurre entre la medición del inventario y el despacho de la nueva orden. Esto incentiva al distribuidor a disminuir sus niveles de inventarios, puesto que en definitiva éstos actúan como un sustituto de la información. De esta manera tienden a aumentar en las frecuencias de los despachos de las mercaderías. Si además se asume que existen economías de escala en los despachos, entonces se deduce que el tamaño óptimo del local debe ser mayor. Por ejemplo, si un local permanece del mismo tamaño y al mismo tiempo duplica la frecuencia de sus pedidos de mercadería como consecuencia de los avances tecnológicos recién mencionados, entonces los camiones sólo debieran llenarse hasta la mitad, con lo que no se estarían aprovechando las economías de escala en el transporte. Se genera entonces un incentivo para agrandar los locales de venta.

\section{Causas de la demanda en el incremento de los hipermercados}

El fuerte aumento de los locales con formato de hipermercado también está motivado por razones de demanda. Los consumidores buscan reducir el tiempo destinado a comprar, lo que los ha llevado a querer adquirir la mayor cantidad de bienes posible en una misma ocasión y en un mismo local, lo que se conoce como one stop shopping.

La intención de los consumidores de reducir el tiempo de compra se debe a que éste se ha ido transformando en un recurso cada vez más escaso. La creciente participación de la mujer en la fuerza laboral parece ser la principal causa de esta tendencia. En Estados Unidos la participación de la mujer en la fuerza laboral medida como porcentaje de la población femenina de 16 años y más, ha ido en constante aumento: 43,3\% en 1970, 
51,5\% en 1980, 57,5\% en 1990 y 59,9\% en 2000 (Bureau of Labor Statistics, USA). El aumento del ingreso per cápita de la población es otro factor que actúa en el mismo sentido, pues aumenta la demanda de oportunidades para disfrutar de ocio. Con el fin de disminuir el tiempo dedicado a actividades que lo usan como insumo, se pretende que las compras de supermercados también se puedan transformar en una entretención o pasatiempo.

Demanda por variedad. Betancourt y Gautschi (1990) desarrollan un modelo formal para explicar la existencia de una demanda por variedad, que es lo que está detrás del deseo del one stop shopping. Ellos afirman que los distribuidores ofrecen una variedad de productos, entre ellos algunos parecen sustitutos, otros complementarios y otros independientes en consumo, de acuerdo a los criterios tradicionales. Lo interesante es que los consumidores desean comprar al mismo tiempo y en el mismo lugar no sólo los bienes considerados tradicionalmente complementarios (pollo y arroz), sino también los sustitutos (pollo y carne) y los independientes (pollo y calcetines). La causa de esto es que aun cuando los bienes pueden ser sustitutos o independientes en su consumo, son complementarios en la compra. Es decir, la demanda por variedad es una consecuencia directa del hecho de que los bienes sean complementarios en la compra. A su vez, esta complementariedad surge debido a la existencia de costos asociados directamente al hecho de comprar.

Costos asociados al hecho de comprar. Los autores identifican los siguientes costos asociados al hecho de comprar:

- el tiempo destinado a comprar;

- la distancia del consumidor al lugar de compra;

- el ajuste que pueda tener que hacer el consumidor en su compra si no encuentra exactamente el producto en la forma y marca que buscaba;

- los costos de información respecto de los productos por comprar;

- el almacenaje de los productos comprados;

- los costos psicológicos de comprar, como pueden ser las molestias causadas por el ruido, la suciedad, etc.

Servicios de distribución. El retailer conoce la existencia de estos costos de compra para los consumidores y busca reducir al menos alguno de ellos como una forma de atraerlos. Esto lo lleva a ofrecer servicios de distribución. 
Los autores identifican los siguientes servicios de distribución:

- la variedad de productos, orientada principalmente a reducir el tiempo de compra de los consumidores;

- la accesibilidad al local, que disminuye la distancia entre los consumidores y el lugar de compra;

- el ambiente del local busca aminorar los costos psicológicos de la compra;

- la disponibilidad de información y probabilidad de obtener el producto deseado en la forma deseada o en el momento deseado, lo que ayuda a restringir los costos de ajuste que deben hacer los consumidores en caso de no encontrar los productos en la forma y marca exactas que ellos requieran.

Tipos de locales. Cada retailer ofrece una combinación diferente de servicios de distribución, pues le resulta muy costoso ofrecerlos todos al mismo tiempo. Esto da como resultado la existencia de diferentes tipos de locales. Por ejemplo, los hipermercados, orientados a ofrecer una gran variedad de productos y así reducir el tiempo destinado a comprar; los locales de conveniencia, que ofrecen poca variedad pero gran accesibilidad al local, puesto que se ubican cerca de los consumidores y tienen horarios de atención muy extensos; y los locales de especialidad, donde su principal característica es ofrecer un alto grado de profundidad en su mercado específico para satisfacer la necesidad del consumidor de encontrar el producto deseado en la forma y en el momento deseados.

Se deduce de este análisis que el fuerte aumento de los formatos tipo hipermercados responde al aumento de la demanda por variedad de parte de los consumidores, la que a su vez se debe al aumento del costo de su tiempo. En otras palabras, los bienes se han ido haciendo cada vez más complementarios en la compra.

\section{Los casos Wal-Mart, Tesco y D\&S}

En esta sección se revisa el caso de Wal-Mart en Estados Unidos, el de Tesco en Gran Bretaña y el de D\&S en Chile. Se detectan patrones comunes: fuerte crecimiento de los formatos tipo hipermercados, aumento de la participación de mercado dentro de la industria, política de precios bajos y clara diversificación de formatos, según los nichos del mercado. 


\subsection{El caso Wal-Mart}

El auge de los hipermercados ha sido liderado en Estados Unidos por la empresa distribuidora Wal-Mart. Esta empresa, a fines de la década de los ochenta, incorporó el rubro alimentos dentro de sus ventas, inspirada en el éxito de estos centros de venta en Europa, donde los hipermercados existían desde mucho antes. La medida fue muy cuestionada inicialmente por los analistas, quienes la consideraban extraordinariamente riesgosa, debido a que el margen de ganancias de los alimentos es tradicionalmente inferior al del resto de los productos. Sin embargo, el tiempo se encargó de demostrar lo contrario: Wal-Mart es hoy día la empresa más grande del mundo, con ventas de US\$ 245 mil millones al año y con 1,4 millones de empleados, lo que lo sitúa como el empleador más grande del mundo. Esta estrategia se implementó en su momento pensando en la sinergia entre los negocios de alimentos y del resto de los productos, como vestuario, jardinería, zapatería, etc., ya que los primeros debían actuar como generadores de tráfico, aumentando así la frecuencia de visitas al local y atando la compra de los otros bienes, que son los que aportan más margen a la empresa. Sin embargo, la realidad excedió a lo planeado y el gran éxito de estas tiendas tipo hipermercados (llamadas supercenters), más allá de significar un enganche de los consumidores, fue el hecho de que los alimentos fueron un gran negocio en sí mismos, puesto que Wal-Mart incorporó anticipadamente los cambios tecnológicos (Yoffie y Wang, 2002).

La estrategia de precios de Wal-Mart ha sido vender siempre a precios bajos: every day low prices (EDLP). Comparaciones de canastas idénticas de productos entre Wal-Mart y algunos competidores arrojan diferencias de precio promedio de alrededor de $25 \%$ (véase Cuadro $\mathrm{N}^{\circ} 3$ ).

CUADRO Nº 3: $\quad$ PRECIOS DE CANASTAS IDÉNTICAS (2002)

\begin{tabular}{lcccccccc}
\hline & Wal-Mart & $\begin{array}{c}\text { Prom. } \\
\text { resto }\end{array}$ & Albertstons & Kroger & Safeway & WinnDixie & Publix & $\begin{array}{c}\text { Ahorro \% } \\
\text { en Wal-Mart }\end{array}$ \\
\hline Las Vegas & 88,4 & 125,2 & 125,9 & 117,6 & 131,9 & & & \\
Tampa & 87,3 & 110,1 & 118,4 & & & 107,6 & 104,3 & $20,7 \%$ \\
Dallas & 89,3 & 118,8 & 122,9 & 118,4 & 115,2 & & & $24,8 \%$ \\
Promedio & 88,3 & 118,0 & & & & & & $25,2 \%$ \\
\end{tabular}


Respecto de los formatos de los locales de ventas, Wal-Mart fue diversificando sus tiendas a través del tiempo con el objeto de abarcar diferentes nichos. En la actualidad cuenta con: tiendas de descuento, hipermercados, supermercados de barrio y los llamados Sam's Clubs (que venden a los consumidores productos al por mayor y por lo tanto a menores precios). Las tiendas de descuento (discount stores) son las más antiguas y no incluyen los alimentos entre sus productos. Los hipermercados (supercenters) sí los incluyen y son también los más exitosos. Su tamaño va desde 17.500 hasta 19.500 metros cuadrados, donde los alimentos ocupan cerca de un tercio del hipermercado. Están generalmente ubicados en áreas suburbanas. Los supermercados de barrio (neighborhood markets), cuyo tamaño es de unos 4.200 metros cuadrados, son la última innovación en cuanto a formato; su tamaño es equivalente al de los supermercados antiguos y están diseñados para "rellenar" las áreas entre los hipermercados o situados en lugares de mucha densidad de población, donde sería impracticable establecer un hipermercado. A fines de 2002, Wal-Mart contaba con un total de 4.414 locales en todo el mundo, 1.170 locales internacionales, 31 supermercados de barrio, 500 Sam's Clubs, 1.066 hipermercados y 1.647 tiendas de descuento (Cuadro $\mathrm{N}^{\circ} 4$ ). El principal aumento en el número de locales viene dado por los hipermercados y por los locales internacionales.

CUADRO N ${ }^{\circ}: \quad$ NÚMERO DE LOCALES DE WAL-MART

\begin{tabular}{lrrr}
\hline & 1992 & 1997 & 2002 \\
\hline Locales en Estados Unidos: & & & \\
$\quad$ Tiendas de descuento & 1.714 & 1.960 & 1.647 \\
Hipermercados & 10 & 344 & 1066 \\
Sam's Clubs & 208 & 436 & 500 \\
Supermercados de barrio & - & - & 31 \\
Locales internacionales & - & 314 & 1.170 \\
Total & 1.932 & 3.054 & 4.414 \\
\hline
\end{tabular}

Fuente: Bell y Feiner: "Wal-Mart Neighborhood Markets", 2003.

El crecimiento promedio anual de las ventas de Wal-Mart es de 17,4\% entre los años 1992 y 2002. La utilidad neta se ha situado en torno al $3 \%$ de las ventas y el retorno sobre patrimonio ha sido algo superior al $20 \%$ (Cuadro $\mathrm{N}^{\circ} 5$ ). 
CUADRO $\mathrm{N}^{\circ}$ 5: $\quad$ WAL-MART: VENTAS Y UTILIDADES

(millones de dólares y porcentajes)

\begin{tabular}{lrrr}
\hline & 1992 & 1997 & 2002 \\
\hline & & & \\
Ventas (mill. US\$) & 43.887 & 104.859 & 217.799 \\
Crecimiento de las ventas netas (\%) & 35 & 12 & 14 \\
Utilidad neta (mill. US\$) & 1.609 & 3.056 & 6.671 \\
Ut. neta sobre ventas (\%) & 3,7 & 2,9 & 3,1 \\
Retorno sobre patrimonio (ROE) (\%) & 26,0 & 19,2 & 20,1 \\
\end{tabular}

Fuente: Bell y Feiner: "Wal-Mart Neighborhood Markets", 2003.

Wal-Mart es la empresa con mayor participación de mercado dentro de Estados Unidos en cuanto al volumen de ventas. Los supermercados de barrio y los Sam's Clubs son comparables a los supermercados tradicionales. En el Gráfico $\mathrm{N}^{\circ} 1$ se observa que en Estados Unidos, respecto de los nueve principales supermercados tradicionales, estos dos formatos de WalMart reunían en 2001 una participación de mercado de 26,5\%; les seguían The Kroger Co. con 17,8\% y en tercer lugar se ubicaba Albertson's con $13,6 \%$.

GRÁFICO N 1: PARTICIPACIÓN DE LOS 9 MAYORES SUPERMERCADOS TRADICIONALES (2001)

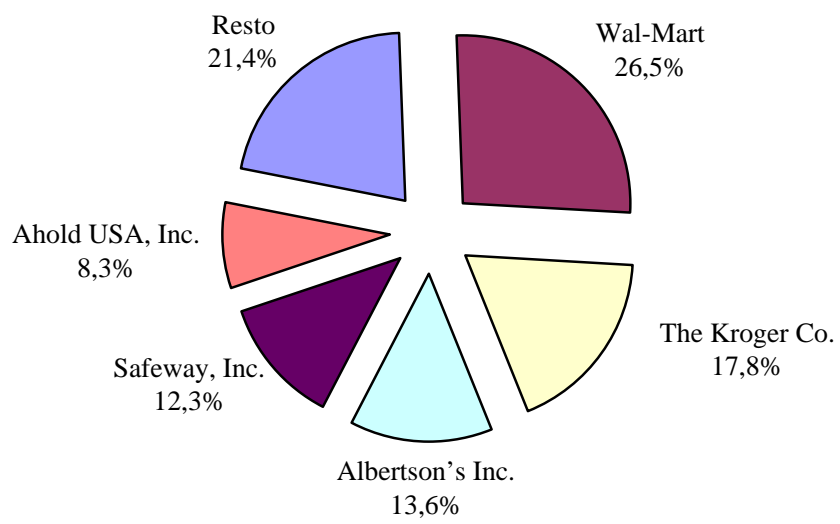

Fuente: Bell y Feiner: "Wal-Mart Neighborhood Markets”, 2003. 
La participación en el mercado por concepto de ventas de los hipermercados (supercenters) de Wal-Mart es aun mayor. En 2001, entre los cinco principales hipermercados, Wal-Mart era, lejos, el más grande, con $72,4 \%$ de las ventas totales, seguido por Kroger con 12,4\% (Cuadro $\mathrm{N}^{\circ} 6$ ).

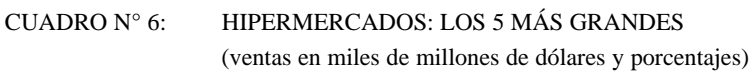

\begin{tabular}{lcr}
\hline & $\begin{array}{c}2001 \mathrm{E} \\
\text { m mill US } \$\end{array}$ & $\%$ \\
\hline Wal-Mart & 64,9 & 72,4 \\
Kroger & 11,1 & 12,4 \\
Meijer & 5,4 & 6,0 \\
Kmart & 6,4 & 7,1 \\
Target & 1,9 & 2,1 \\
& & 100,0 \\
Total & 89,7 & \\
\hline
\end{tabular}

Fuente: Bell y Feiner: "Wal-Mart Neighborhood Markets", 2003.

La expansión internacional de la empresa, que comenzó en 1993, no ha sido fácil. Los esfuerzos de internacionalización se han centrado en los mercados europeos, debido a su alto poder adquisitivo. Wal-Mart ha entrado también en Asia y en el resto de América. Se estableció en Asia: en Corea, con utilidades inferiores a las esperadas; en China, donde ha debido ir sorteando las restricciones estatales; en Japón, donde, tras fracasar en 1997, ha vuelto a entrar en 2002; y en Indonesia, con el peor resultado de todos. En el mercado del resto de América ha tenido éxito en Canadá y México, países donde ha pasado a ser el retailer dominante. También ha entrado en Brasil, de manera muy agresiva, y en Argentina, con dificultades.

\subsection{El caso Tesco}

Tesco es la empresa que lidera este cambio en la industria de los supermercados en Gran Bretaña. En 1997, Tesco desarrolló un agresivo plan a diez años, según el cual se establecían dos metas principales. Una era participar fuertemente tanto en el rubro de los alimentos como en los de no alimentos (vestuario, música, y otros). La otra fue la internacionaliza- 
ción. Las cifras cinco años después demostraban el éxito del plan: en 2002 Tesco alcanzó un 4\% de participación en el mercado total de los no alimentos. Respecto del tamaño alcanzado, la empresa contaba con 1.023 locales: 750 en Gran Bretaña y el resto en diez países. Los principales competidores internos son Asda (adquirida por Wal-Mart) y Morrison. Le siguen Sainsbury's y Safeway. Los locales internacionales representan cerca del $42 \%$ del espacio total de venta, pero sólo un $10 \%$ de sus ingresos operacionales. Ahí, Carrefour y Wal-Mart son los principales competidores. La estrategia de precios es la de precios bajos siempre (EDLP). La diversificación de formatos es la siguiente: los metro, pequeños locales (190 metros cuadrados) ubicados en el centro de la ciudad y orientados a los sandwiches y comida preparada; los express, locales también pequeños (900 metros cuadrados) ubicados generalmente cerca de las bombas de bencina Esso. Los superstore (3.200 metros cuadrados), que son los tradicionales supermercados Tesco, y los extra (8.400 metros cuadrados), que son los de formato hipermercado y a través de los cuales se abarca el rubro de los no alimentos (Bell 2003).

\subsection{El caso D\&S}

En Chile, la cadena de supermercados D\&S es la que lidera el mercado con una participación de ventas de $34,4 \%$ a mayo de 2004. Le sigue Cencosud con 21,4\%. Muy por debajo se sitúan San Francisco', con $3,2 \%$, y Unimarc, con $3,1 \%$ tras una pérdida de participación de mercado sostenida en el tiempo (ver Cuadro $\mathrm{N}^{\circ} 7$ ).

En 2003, D\&S adoptó la estrategia de "precios bajos siempre"; antes tenía la política de promociones de fines de semana y de productos en particular. Jumbo, el segundo actor, ha seguido una política diferente de precios basada en promociones puntuales de diferentes productos y en la calidad; de ahí su lema: "La calidad no cuesta más".

Las ventas de D\&S han crecido a una tasa promedio de $9 \%$ desde 1998 hasta 2003. El margen de operación se sitúa en torno al 22\% (22,5\% en 2003) y la utilidad neta en $2,3 \%$ en el período ( $1,8 \%$ en 2003$)$. La rentabilidad sobre el patrimonio se situó el último año en 6,9\% (Cuadro $\mathrm{N}^{\circ} 8$ ).

La diversificación de formatos ha seguido la pauta internacional: los Líder Mercado, que son hipermercados con $9.600 \mathrm{~m}^{2}$ en promedio; los Líder Vecino, con $3.800 \mathrm{~m}^{2}$ en promedio, que equivalen a los supermerca-

${ }^{1}$ San Francisco fue adquirida por Falabella en julio de 2004. 
CUADRO N ${ }^{\circ}$ 7: INDUSTRIA DE LOS SUPERMERCADOS:

PARTICIPACIÓN DE MERCADO EN PORCENTAJES

\begin{tabular}{lrrc}
\hline & 1996 & 2000 & 2004 mayo \\
\hline D\&S & 19,2 & 28,3 & 34,4 \\
Cencosud & 6,3 & 7,0 & 21,4 \\
Santa Isabel & 13,3 & 10,3 & 3,1 \\
Unimarc & 8,5 & 5,4 & 3,2 \\
San Francisco & 2,0 & 2,7 & 2,7 \\
Montserrat & 4,2 & 3,2 & 2,7 \\
Montecarlo & 1,8 & 2,0 & 3,1 \\
Carrefour & & 1,6 & 29,4 \\
Rendic & & 2,2 & 100 \\
Las Brisas/Extra & 4,1 & 2,5 & \\
Resto & 40,6 & 34,9 & \\
& & $100^{1}$ & \\
Total & 100 & & \\
\hline
\end{tabular}

Notas:

${ }^{\text {a }}$ D\&S compra Carrefour en 2003.

${ }^{\mathrm{b}}$ Cencosud compra Santa Isabel y Tops en 2003 y Las Brisas/Extra en 2004.

Fuente: Asociación Gremial de Supermercados de Chile (Asach): www.asach.com

CUADRO N ${ }^{\circ} 8: \quad$ D\&S: PRINCIPALES DATOS FINANCIEROS

\begin{tabular}{lrrrrrr}
\hline & 1998 & 1999 & 2000 & 2001 & 2002 & 2003 \\
\hline & & & & & & \\
Ventas (mill. \$ dic. 03) & 664.373 & 833.517 & 779.717 & 854.084 & 939.270 & 1.023 .654 \\
Margen de explotación (ingresos) & 21,1 & 20,9 & 21,8 & 22,5 & 22,8 & 22,5 \\
Resultado operacional (\% ingresos) & 3,7 & 3,3 & 5,7 & 6,0 & 4,0 & 3,9 \\
Utilidad neta (\% ingresos) & 2,8 & $-1,1$ & 3,6 & 4,3 & 2,3 & 1,8 \\
Rentabilidad sobre patrimonio (\%) & 7,4 & $-3,9$ & 11,9 & 14,6 & 8,0 & 6,8 \\
\hline
\end{tabular}

Fuente: D\&S: www.dys.cl

dos tradicionales, y los Líder Express, los más pequeños, con $1.500 \mathrm{~m}^{2}$ (véase Cuadro $\mathrm{N}^{\circ}$ 9). Actualmente, el $84 \%$ del total de las ventas de D\&S se realiza a través de hipermercados (Líder Vecino + Líder Mercado). Las ventas de artículos no alimentos representan hoy el $26,4 \%$ del total de las ventas de D\&S. 


\begin{tabular}{lcc}
\hline & $\mathrm{m}^{2}$ promedio & $\mathrm{N}^{\circ}$ de locales \\
\hline Supermercados & 1.532 & 26 \\
Líder Express & & \\
Hipermercados & 6.987 & 42 \\
Líder Vecino & 3.821 & 19 \\
Líder Mercado & 9.603 & 23 \\
Total & 4.902 & 68 \\
\hline
\end{tabular}

Fuente: D\&S: www.dys.cl

\section{Relaciones entre proveedores y distribuidores en la industria de los supermercados}

Una de las consecuencias directas de la concentración de la industria de supermercados es el desplazamiento de poderes desde proveedores hacia distribuidores.

\subsection{Marco conceptual}

Desde el punto de vista económico, la distribución es el último eslabón en la cadena de producción. El óptimo para el productor es tratar de ejercer el máximo control sobre toda la cadena productiva, de manera de conseguir el resultado que él estima conveniente. Desde esta perspectiva, el productor intentará también controlar la distribución de sus productos. Para esto evaluará como primera opción la posibilidad de verticalizar su producción, y lo hará siempre y cuando los beneficios de hacerlo sean mayores que los costos. Dado que el productor no es capaz, por lo general, de distribuir él mismo sus productos, los entrega a terceros (los distribuidores) para que hagan esta tarea. Sin embargo, trata de controlar lo más posible a los distribuidores de manera de inducirlos a actuar de la forma que a él más le conviene. Esto lleva al surgimiento de negociaciones contractuales entre ambos agentes. En la literatura dichas negociaciones reciben el nombre de restricciones verticales (Carlton y Perloff, 20001, p. 396). Entre las restric- 
ciones impuestas por los proveedores sobre los distribuidores se pueden mencionar, por ejemplo: precio mínimo de venta al público, la venta de un número mínimo de unidades al público y la imposición de no vender determinados productos de la competencia. De esto se desprenden al menos dos conclusiones: la primera es que las relaciones entre proveedores y distribuidores son por definición complicadas, y la segunda, que la capacidad que tengan los proveedores de ejercer restricciones verticales sobre los distribuidores dependerá de las formas en que se organicen ambas industrias.

Lo que ha sucedido en el mundo es que la industria de la distribución se ha ido concentrando a raíz de los cambios comentados en la sección anterior. Junto con esto, esta industria ha dado señales de fuerte competencia, lo que se refleja en las agresivas políticas de precios bajos para los consumidores. La mayor concentración en el mercado de la distribución ha traído como consecuencia un profundo cambio en las relaciones con los proveedores. Mientras más concentrada sea la industria de la distribución, más difícil es para los proveedores imponerles restricciones verticales. Y al revés. Teóricamente este aumento de la concentración de los distribuidores genera un desplazamiento de poderes desde proveedores hacia distribuidores, ceteris paribus (esto es, siempre y cuando no se tienda a producir también una concentración en las industrias productivas). Si a esto se suma el hecho de que esta industria muestra fuertes signos de competencia, entonces los beneficios que los distribuidores logren obtener de sus negociaciones con los proveedores, como consecuencia de su mejor posición relativa frente a ellos, son traspasados a los consumidores. En esta línea, Dobson y Waterson (1997) afirman que si la industria de la distribución se concentra y los consumidores visualizan como fuertes sustitutos los servicios que entregan las distintas cadenas de retail, entonces los precios finales de venta al público tienden a bajar como consecuencia de dicho aumento de concentración. Esto se dará incluso en el caso límite en que existan sólo dos cadenas de distribución, si ellas compiten a la manera de Bertrand $^{2}$ y si sus servicios son percibidos como perfectos sustitutos por los consumidores. Así, en estas situaciones, la disminución del poder de los proveedores como consecuencia del aumento en la concentración de los distribuidores puede ser suficiente para inducir una caída en los precios finales, con lo que los consumidores se verían beneficiados.

${ }^{2}$ El modelo de Bertrand supone que las empresas compiten entre sí a través de los precios, lo que lleva a un resultado competitivo. 


\subsection{La evidencia empírica}

De los análisis empíricos realizados a nivel internacional no se ha podido confirmar la hipótesis teórica de que se haya producido un desplazamiento de poder desde los proveedores hacia los distribuidores. El desplazamiento de poder se mide a través de las rentabilidades relativas de los retailers en relación con los productores o proveedores.

Farris y Ailawadi (1992) fueron los primeros en poner en duda este desplazamiento de poderes desde productores de productos empaquetados hacia retailers. Ellos analizaron las tendencias en las utilidades de productores y distribuidores y observaron que éstas no eran concordantes con dicho desplazamiento de poder. Sin embargo, este resultado se obtuvo para los grandes productores y no necesariamente para los pequeños. Esto sugiere que el tamaño de las empresas puede ser un factor importante en la habilidad para trabajar con un retailer poderoso. Messinger y Narasimhan (1995) también concluyen que no existe evidencia de un desplazamiento de poder desde proveedores hacia distribuidores. Bloom y Perry (2001) detectaron que aquellos productores que vendían a retailers poderosos no mostraban necesariamente menores utilidades que aquellos que no. Ellos afirman que los grandes proveedores que fueron capaces de desarrollar una buena relación con Wal-Mart pueden tener un mejor desempeño financiero y extraer más utilidades de su mercado que sus contrapartes que no desarrollaron tal vínculo. Así, para los proveedores es conveniente establecer buenas relaciones con Wal-Mart, ya que, a pesar de tener que cumplir con grandes exigencias en precios y tiempos de despacho, entre otros, saben que acceden a aumentar su participación en el mercado. Un proveedor decía: "Sólo hay una cosa peor que venderle a Wal-Mart, no venderle a Wal-Mart". Uno de los elementos que ha beneficiado a las relaciones entre Wal-Mart y sus proveedores ha sido la implemetación del retail link. Este sistema informa computacionalmente a los proveedores los datos relevantes de cada uno de los puntos de venta de Wal-Mart. Con esto se logra una mayor eficiencia en el manejo de inventarios y despacho de mercadería y a la vez les permite a los productores estimar las ventas a futuro, todo esto tienda por tienda. El costo de desarrollo y perfeccionamiento de este sistema fue altísimo (se estima que Wal-Mart invirtió cerca de US\$ 4.000 millones y los proveedores también debieron realizar una inversión sustancial) y es hoy en día uno de los más sofisticados del mundo en la materia (Ghemawat, Mark y Bradley, 2003).

Una de las posibles explicaciones para la falta de evidencias del desplazamiento de poderes desde proveedores hacia supermercados es que 
la creciente concentración de esta industria ha significado un cambio estructural mayor a lo largo de la cadena completa del proceso productivo y no sólo del comercio detallista. De acuerdo con esto, los proveedores han ido ajustándose a esta nueva organización de los distribuidores y han aumentado la eficiencia de sus procesos productivos, lo que en definitiva les ha permitido mantener o incluso incrementar sus rentabilidades. Otra de las explicaciones que se tienden a dar es que el aumento en la concentración de los supermercados ha inducido a que los proveedores se concentren aún más, con lo que no se cumple la premisa básica del desplazamiento de poderes. Messinger y Narasimhan (1995) señalan que, en Estados Unidos, la concentración de la industria de la distribución ha sido de la misma magnitud que la concentración de la industria manufacturera a nivel nacional. Por último, se podría pensar que efectivamente se han producido desplazamientos de poder a lo largo de la cadena productiva, pero que éstos no se han visto reflejados en aumentos de las rentabilidades de los distribuidores, ya que se han traspasado todos los beneficios de las negociaciones a los consumidores, debido a la fuerte competencia de esta industria.

\section{Marcas propias}

Las marcas propias son aquellas cuyo dueño es el intermediario o distribuidor del producto y no necesariamente su fabricante, quien no se identifica con la marca.

Las marcas propias nacieron en el siglo XIX, como una idea de los distribuidores de producir ellos mismos sus productos o bien de comprarlos directamente a los productores para evitarse los costos de intermediación. Las primeras marcas propias se dieron para el vestuario y de ahí se siguió a los alimentos.

El concepto del distribuidor dueño de la marca propia era vender siempre a precios más bajos que las marcas tradicionales, de manera de ofrecer una alternativa barata a los consumidores. La idea era capturar un nicho de mercado inexplotado hasta ese momento, el de menores precios e inferior calidad. Dadas estas características, sus ventas fluctuaban inversamente al ciclo económico hasta las décadas de los setenta u ochenta, época en la que todavía el mercado de la distribución se encontraba altamente fragmentado. En la década de los noventa se genera un nuevo impulso en el desarrollo de las marcas propias. De acuerdo al Boston Consulting Group (2003), la participación de las marcas propias dentro del total de las ventas de abarrotes para un conjunto de países europeos y Estados Unidos creció en forma importante entre 1997 y 2002 (Cuadro $N^{\circ} 10$ ). 


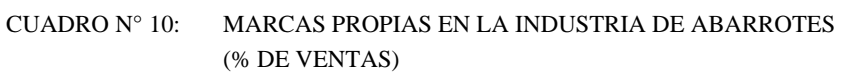

\begin{tabular}{lcc}
\hline & 1997 & 2002 \\
\hline Gran Bretaña & 29,7 & 39,0 \\
Bélgica & 25,8 & 26,9 \\
Alemania & 11,3 & 24,0 \\
Francia & 16,8 & 20,8 \\
Holanda & 17,8 & 18,6 \\
España & 16,2 & 17,6 \\
Estados Unidos & 14,1 & 15,6 \\
Italia & 8,0 & 11,1 \\
\hline
\end{tabular}

Fuente: Brady, Brown y Hulit: "Private Label: Threat to Manufacturers, Opportunity for Retailers", 2003.

\subsection{Causas del fuerte desarrollo de las marcas propias}

Tras el fuerte desarrollo de las marcas propias es posible encontrar causas tanto de oferta como de demanda.

\subsubsection{Causas de oferta}

La concentración de la industria de la distribución se cuenta como una causa importante del fuerte incremento de las marcas propias en el mundo en las últimas dos décadas (Putsis y Coterill, 1999). Las marcas propias comienzan a ser vistas como un aspecto importante en la generación de utilidades del negocio. Se distinguen tres vías por las que las marcas propias agregan rentabilidad al negocio:

a) Representan una fuente directa de ingresos. Las marcas propias constituyen una fuente directa de ingresos para los distribuidores en relación con la venta de otras marcas. El margen de venta que el distribuidor obtiene de una marca propia es mayor que el que obtiene de una marca tradicional (Ailawadi, 2001; Ailawadi y Harlam, 2004). Existen al menos dos posibles razones para esto: los proveedores de marcas propias tienen menor poder de mercado que los proveedores de marcas tradicionales, puesto que su grado de concentración es menor y operan en un mercado competitivo donde no existe diferenciación de productos, por lo que sus precios de venta a los distribuidores debieran estar cerca de sus costos marginales (Ailawadi y Harlam, 2000). Por otra parte, significan un ahorro 
de costos respecto del ítem publicidad. Para el caso de las marcas tradicionales, la inversión requerida en publicidad debe efectuarse producto por producto, lo que representa un factor de costo importante. En cambio, en el caso de las marcas privadas se publicita la marca del supermercado y esto abarca toda la gama de productos que éste quiera incluir. Ambos factores juntos hacen que el costo de una marca propia para el distribuidor sea menor que el de una marca tradicional. A pesar de lo anterior, los estudios advierten del peligro de desplazar las marcas tradicionales por las marcas propias, pues esto puede repercutir negativamente en las utilidades del distribuidor. La coexistencia de marcas propias junto con marcas tradicionales es clave en la rentabilidad del distribuidor (Ailawadi y Harlam, 2000). Existe una demanda por variedad de parte de los consumidores, que es del interés de los distribuidores respetar. Así, las empresas distribuidoras orientadas a obtener sus utilidades por la vía de generar un alto tráfico de público, deben ofrecer una amplia y profunda variedad de productos. Deben, entonces, mantener un adecuado balance entre marcas propias y marcas tradicionales (Dhar y Hoch, 1997). Otro enfoque que lleva a la misma conclusión se refiere a que dado que las marcas propias se venden a precios a veces muy inferiores que los de las marcas tradicionales, éstas pueden terminar reportando una menor rentabilidad que las tradicionales, aun cuando su margen de venta sea mayor (Ailawadi y Harlam, 2004).

b) Permiten que los distribuidores se diferencien entre sí. A partir de la década de los noventa en Estados Unidos, y a medida que se va concentrando la industria del retail, se comienza a promover la cadena de distribución como una marca. La mayor lealtad a una determinada cadena o su diferenciación repercute positivamente en las utilidades del retailer. Las marcas propias pasan a ser una vía eficiente por la cual los distribuidores logran diferenciarse de sus competidores y conseguir fidelidad de parte de los consumidores. En efecto, Corstjens y Lal (2000) muestran que la lealtad a la tienda derivada del consumo de una marca propia de calidad puede aumentar las utilidades del distribuidor, aun cuando el margen de ganancias de ésta no sea mayor que el de las marcas tradicionales. Se ha demostrado empíricamente que los consumidores que compran al menos algunos productos de marcas propias contribuyen más a la rentabilidad de los distribuidores, en comparación con aquellos que sólo demandan marcas tradicionales. Sin embargo, se ha visto que los consumidores que están muy enfocados a las marcas propias reportan menos rentabilidad, pues se trata en general de clientes muy selectivos al momento de comprar, que adquieren muy pocos bienes y sólo los de menores precios (Ailawadi y Harlam, 2004). 
c) Aportan en el desplazamiento de poder desde los proveedores hacia los distribuidores. Desde un punto de vista teórico, las marcas propias desplazan poder de negociación desde los proveedores hacia los distribuidores. Narasimhan y Wilcox (1998) afirman que cuando las preferencias de los consumidores por las marcas tradicionales no son fuertes y ellos se muestran dispuestos a cambiarse a una marca propia, entonces los distribuidores se beneficiarán por la introducción de éstas, puesto que lograrán obtener menores precios de compra de parte de los proveedores de marcas tradicionales. Sin embargo, sus investigaciones empíricas aportan sólo un leve sustento a tal afirmación. En Estados Unidos existe evidencia de que la probabilidad de que un distribuidor introduzca una marca propia en una determinada categoría de productos es mayor mientras mayor sea la participación de la marca líder en esa categoría. Esto lleva a concluir que los distribuidores estarían buscando aumentar su poder de negociación con el proveedor de dicha marca a través de la presencia de las marcas propias (Morton y Zettelmeyer, 2000).

Los incentivos de los distribuidores para proveer marcas propias llevan a que se produzca una verticalización hacia atrás del proceso productivo. En la sección anterior se mencionaba que los productores, como primera opción, siempre buscan manejar directamente cada una de las etapas de su proceso productivo. Es decir, siempre que sea eficiente hacerlo, el productor tratará de integrar verticalmente lo más posible su negocio. Se mencionaba también que el último eslabón en el proceso productivo era la distribución y que ésta era claramente inviable de verticalizar, por lo que el productor debía entregar tal tarea a terceros. Aquí, por todas estas razones, se obtiene una cierta tendencia a que se dé el proceso al revés. Es decir, es el distribuidor quien termina por controlar al menos parte del proceso productivo.

\section{1.2. Causas de demanda}

Los consumidores actuales presentan algunos aspectos nuevos en su comportamiento: son consumidores más informados y han madurado sus hábitos de compra. Esta nueva conducta implica que están más capacitados para entender mejor las relaciones de calidad y precio de los bienes, lo que en definitiva los lleva a ser más abiertos a adquirir productos y marcas nuevas, como es el caso de las marcas propias, y menos leales a las marcas tradicionales. Éste es en definitiva un escenario propicio para la introducción de marcas propias (Fiske y Silverstein, 2002). 


\section{Conclusiones}

De este trabajo se desprende que el sector distribución ha experimentado profundos cambios en el mundo a partir de la década de los ochenta. Las principales características de estos cambios son la concentración de la industria, el auge de los hipermercados y las políticas de precios bajos para los consumidores finales. Los avances tecnológicos en el campo de la informática han permitido optimizar los despachos de mercaderías y manejo de inventarios, generando así economías de escala y de ámbito en la industria de la distribución. Estudios sobre la productividad marginal de los factores (PTF) en Estados Unidos muestran que el sector retail es el que presenta un mayor crecimiento de esta variable en el período 19952000. Esto ha favorecido a la concentración de la industria de la distribución, ya que las empresas que adoptan oportunamente los adelantos tecnológicos pueden ofrecer menores precios debido al ahorro de costos y van ganando así participación de mercado respecto de sus competidores. Los adelantos tecnológicos han permitido también el auge de los hipermercados. En este último caso también se encuentra como causa la creciente participación de la mujer en el mercado laboral, pues aumenta la demanda por el one stop shopping a raíz del aumento del costo del tiempo que esto conlleva.

La concentración de la industria de la distribución produce un desplazamiento de poderes desde los proveedores hacia los distribuidores. Si la industria de la distribución es competitiva, entonces los beneficios de las mejores negociaciones obtenidas con los proveedores deberán ser traspasados a los consumidores. La evidencia empírica internacional no permite afirmar que efectivamente se haya producido tal desplazamiento de poderes, medido como la evolución de diversos índices de rentabilidades relativas entre proveedores y distribuidores. Entre las explicaciones a estos hallazgos se encuentran los posibles aumentos de concentración de las industrias proveedoras - por lo que no habría tal desplazamiento de poder-, los aumentos de eficiencia de los proveedores como consecuencia de las mayores exigencias de los distribuidores y la incapacidad de los distribuidores de retener para ellos los beneficios obtenidos de las negociaciones.

La concentración de la industria favorece también al desarrollo de las marcas propias. Éstas representan, además de una fuente directa de ingresos para los distribuidores, una manera de diferenciarse respecto de sus competidores y una forma de aportar en el desplazamiento de poderes 
desde proveedores de marcas tradicionales hacia ellos. Los consumidores actuales están más proclives a aceptarlas, puesto que su lealtad a las marcas tradicionales ha disminuido como consecuencia de su mayor capacidad de informarse en cuanto a las relaciones calidad-precio de los bienes.

\section{REFERENCIAS}

AcNielsen, Child: "Cambios en el Mercado Chileno 2004: Entendiendo al Consumidor". 2004, p. 5.

Ailawadi, K. L. y B. Harlam: "The effect of Store Brands on the Retailer Profitability: An Empirical Analysis". Tuck School Working Paper, Dartmouth College, 2000.

Ailawadi, K. L.: “The retail power-performance conundrum: What have we learned?" Journal of Retailing 77 (2001), pp. 299-318.

Ailawadi, K. L. y B. Harlam: "An Empirical Analysis of the Determinants of Retail Margins: The Role of Store-Brand Share”. Journal of Marketing, Vol. 68, $\mathrm{N}^{\circ} 1$ (enero 2004).

Asociación Gremial de Supermercados de Chile: www.asach.com

Bell, D.: Tesco Plc. Harvard Business School, March 6, 2003.

Bell, D. y J. Feiner: "Wal-Mart Neighborhood Markets". Harvard Business School, November 22, 2003.

Betancourt R. y D. Gautschi: "Demand, Complementarities, Households Production and Retail Assortments". Marketing Science, Vol. 9, No 2, primavera 1990, pp. 146-161.

Bloom, P. y V. Perry: "Retailer Power and Supplier Welfare: The Case of Wal-Mart". Journal of Retailing, 77 (2001), pp. 379-396.

Brady, L., A. Brown y B. Hulit: "Private Label: Threat to Manufacturers, Opportunity for Retailers". The Boston Consulting Group, 2003. www.bcg.com

Bureau of Labor Statistics (USA): Current Population Survey. U.S. Department of Labor.

Carlton, D. y J. Perloff: Modern Industrial Organization. Addison Wesley Longman, $3^{\mathrm{a}}$ edición, 2000.

Cortjens, M. y R. Lal: "Building Store Loyalty Through Store Brands". Journal of Marketing Research, Vol. 37, №3 (2000), pp. 281- 291.

Dhar, S. y S. Hoch: "Why Store Brand Penetration Varies by Retailer". Marketing Science, Vol. 16, N 3 (1997), pp. 208-227.

Dobson y Waterson: "Countervaling Power and Consumer Prices". The Economic Journal, $N^{\circ} 107$ (marzo 1997), pp. 418-430.

Dobson, P. y M. Waterson: "Retailer Power: Recent Developments and Policy Implications". Economic Policy, Vol. 14, № 28 (1999), p. 135.

D\&S: www.dys.cl

Farris, P. y K. Ailawadi: "Retail Power: Monster or Mouse? "Journal of Retailing, Vol. 68, $\mathrm{N}^{\circ} 4$ (1992), pp. 351-369.

Fernald, J. y S. Ramnath: "The Acceleration in US Total Factor Productivity after 1995: The Role of Information Technology". Economic Perspectives, Vol. 28, N 1 (2004), pp. 52-68.

Fiske, N. y M. Silverstein: "Trading Up: The New Luxury and Why We Need It". The Boston Consulting Group, 2003. www.bcg.com 
Foster, L., J. Haltiwanger y C. J. Krizan: “The Link between Aggregate and Microproductivity Growth: Evidence from Retail Trade". National Bureau of Economic Research, Working Paper $\mathrm{N}^{\circ} 9120$ (2002).

Ghemawat, P., K. Mark y S. Bradley: "Wal-Mart Stores in 2003". Harvard Business School. octubre 23, 2003.

Holmes, T. "Bar Codes Lead to Frequent Deliveries and Superstores". Rand Journal of Economics, Vol. 32, $\mathrm{N}^{\circ} 4$ (invierno 2001), pp. 708-725.

INE (Instituto Nacional de Estadísticas): ine.cl

Messinger, P. y C. Narasimhan: "Has Power Shifted in the Grocery Channel?" Marketing Science, Vol. 2, $\mathrm{N}^{\circ}$ 2, 1995.

Morton, F. y F. Zettelmeyer: "The Strategic Positioning of Store Brands in Retailer-Manufacturer Bargaining”. Working Paper Series. Yale School of Management, abril 2000.

Nakamura, L.: "Is the US Economy Really Growing to Slowly? Maybe We're Measuring Growth Wrong". Business Review, Federal Reserve Bank of Philadelphia, marzo/ abril (1997).

Narasimhan, C. y R. Wilcox: "Private Labels and the Channel Relationship: A Cross-Category Analysis". Journal of Business, Vol. 71, № 4 (1998), pp. 573-601.

Putsis, W. y R. Coterill: "Share, Price and Category Expenditure-Geografic Markets Effects and Private Labels". Managerial and Decision Economics, 20 (1999), pp. 175-187.

Sieling, M., B. Friedman y M. Dumas: "Labor Productivity in the Retail Industry, 1987-99". Monthly Labor Review, Vol. 124, $\mathrm{N}^{\circ} 12$ (diciembre 2001).

Yoffie, D. y Y. Wang: “Wal-Mart in 2002”. Harvard Business School. Marzo 22, 2002. 